\title{
Estrutura etária e crescimento econômico: evidências no Rio Grande do Sul/Brasil (1991-2010)
}

\section{Social structure and economic growth: evidence in Rio Grande do sul/Brazil (1991-2010)}

\author{
LaUana RossetTo LazaretTi * \\ Pascoal José Marion Filho **
}

\begin{abstract}
The aim of this study is to analyze the relationship between the change in the age structure and the economic growth in Rio Grande do Sul from the Demographic Census of 1991, 2000, and 2010. Spatial econometric models are used. The demographic variables were significant for the convergence of per capita income. The increase in population density and the quality of life of the population contribute to economic growth. The absolute population growth lowers per capita income, while the age group of people aged between 35 and 45 years are those that contribute the most to the increase in income.
\end{abstract}

Keywords: demographic transition, economic growth, Rio Grande do Sul.

\section{Resumo}

O objetivo da pesquisa é analisar a relação entre a mudança na estrutura etária e o crescimento econômico no Rio Grande do Sul, a partir dos Censos Demográficos de 1991, 2000 e 2010. Utilizam-se modelos econométricos espaciais. As variáveis demográficas foram significativas para a convergência da renda per capita. $\mathrm{O}$ aumento da densidade demográfica e a qualidade de vida da população contribuem para o crescimento econômico. $\mathrm{O}$ aumento absoluto da população diminui a renda per capita, enquanto a faixa etária de pessoas com idade entre 35 e 45 anos são as que mais contribuem para o aumento da renda.

Palavras-chaves: transição demográfica, crescimento econômico, Rio Grande do Sul.

\footnotetext{
* Pontifícia Universidade Católica do Rio Grande do Sul, correo-e: lauana.lazaretti@yahoo.com.br

**Universidade Federal de Santa Maria, correo-e: pascoaljmarion@yahoo.com.br
} 


\section{Introduçáo}

A partir da segunda metade do século XX, as preocupaçóes populacionais da Organização das Nações Unidas (ONU, 2017) e do Banco Mundial voltaram-se para a explosão demográfica e seus impactos sobre a oferta de alimentos e os recursos naturais. Essa preocupação mudou quando países mais desenvolvidos, como os da Europa, passaram a obter taxas de fecundidade baixa e crescimento populacional em direção a zero. Essa tendência demográfica vem se alastrando para os outros continentes, trazendo consigo repercussóes na estrutura etária e em suas economias (Brito, 2008).

As taxas de natalidade e mortalidade provocam mudanças na estrutura etária da populaçẫo. A redução destas, e o aumento na expectativa de vida dos indivíduos, tendem a aumentar o número de idosos e a diminuir a quantidade de pessoas jovens. O processo possui uma etapa intermediária, em que o número de pessoas em idade ativa chega ao ponto de máximo. Este período é chamado de bônus demográfico, já que há mais pessoas em idade para trabalhar e cria oportunidades de crescimento econômico. O envelhecimento populacional, segundo Lefebvre (2006), pode afetar o crescimento econômico por meio dos efeitos potenciais sobre a poupança, os investimentos, o estoque de capital e o trabalho. Além disso, as mudanças na estrutura etária provocam alteraçóes no consumo agregado das famílias, o que gera impactos sobre os setores e, consequentemente, sobre a produçáo e o crescimento econômico (Prskawetz e Lindh, 2007).

Os estudos que implicam crescimento econômico carecem de uma análise que envolva o aspecto populacional em seus modelos. Segundo Vasconcelos et al. (2008) e Stampe (2013), com base no modelo de crescimento econômico exógeno de Solow (1956), as forças de crescimento econômico foram simplificadas quando analisadas com as relaçóes do crescimento da população. Com isso, outros estudos do crescimento econômico endógeno surgiram, destacando-se Lucas (1988) e Romer (1990). Para esses autores, o capital humano provoca reflexos positivos sobre o crescimento da economia.

No decorrer dos estudos toma forma a importância da estrutura etária da populaçáo sobre o crescimento econômico das Regióes, sobrepondo a ideia da influência apenas do crescimento populacional absoluto. Segundo Bloom et al. (2001), enquanto o crescimento da população provoca efeitos negativos, no crescimento econômico, as mudanças na estrutura etária da população; como a maior participação da população em idade ativa, geram aumento do crescimento econômico. As estruturas etárias se diferenciam entre as Regiôes, e são um fator importante para o crescimento do produto. 
Com isso, em meio ao acelerado processo de envelhecimento populacional brasileiro, o Rio Grande do Sul possui a menor taxa média geométrica anual de crescimento da população, 0.49\% em 2010 (IBGE, 2017). Se comparado com os demais estados brasileiros, o Rio Grande do Sul é caracterizado por um processo de transição demográfica avançado e chegou ao máximo de pessoas em idade ativa em 2016 (69.60\% da população). Desta forma, o objetivo da pesquisa é analisar a relação entre a mudança na estrutura etária e o crescimento econômico no Rio Grande do Sul, a partir dos Censos Demográficos de 1991, 2000 e 2010. Para tanto, utiliza-se suporte econométrico espacial.

Por fim, este trabalho está dividido em cinco seçóes, sendo a primeira delas esta introdução. A segunda seção traz o referencial teórico e a terceira trata dos aspectos metodológicos do estudo. Na quarta seção são apresentados e discutidos resultados da pesquisa, e na última se encontram as consideraçóes finais do estudo.

\section{Estrutura etária e crescimento econômico}

As teorias do crescimento econômico evoluem com o passar do tempo, e a contribuição do crescimento da população foi considerada, por alguns autores, como um ponto negativo para o crescimento do produto. No decorrer da contribuição do pensamento macroeconômico, a estrutura etária passou a ser importante para o aumento do crescimento econômico, já que quanto mais pessoas em idade ativa, maior é a oferta de mão de obra e, consequentemente, a produção aumenta.

Inicialmente, busca-se discutir essas teorias, que partem da visão clássica, passa-se pela tentativa teórica demográfica e para a visão neoclássica, juntas conduzem os estudos recentes, os quais são apresentados posteriormente. Marshall (1996) coloca o homem como o principal instrumento da produção de alimentos. $\mathrm{O}$ aumento da população ocorre quando os nascimentos se sobrepóem aos óbitos, variando entre as regióes devido ao clima, a renda, a cultura e os costumes. Ao comparar o crescimento populacional de alguns países, o autor relatou diferentes estágios de crescimento e características distintas entre eles. A produçáo, a educação, o custo dos filhos, a idade e o tempo para casar possuem discrepâncias. Essas características compóem de diferentes formas o crescimento econômico e populacional das regióes.

Kuznets (1986) trata o crescimento da população como fator indutor do crescimento econômico e do consumo da sua produçáo. Na Europa, o crescimento populacional foi precoce e acompanhado da elevaçáo do desempenho econômico. $\mathrm{O}$ autor cita que o padrão de crescimento po- 
pulacional é diferente na América Latina, Ásia e África, nestes a aceleração populacional inicia mais tarde. Ainda se tratando desses países, uma associação inversa a da Europa é visualizada, pois nos períodos mais antigos o crescimento populacional foi acompanhado por desempenho econômico elevado e crescente. Já em períodos recentes, o crescimento rápido da população foi acompanhado por baixo desempenho econômico.

Neste sentido, Lewis (1969) analisa a oferta de mão de obra em alguns países, e ao tratar da Europa relata que o conjunto de oferta era limitado e o crescimento da economia ocorria de forma automática. Por outro lado, na Ásia a oferta de mão de obra era ilimitada e o crescimento econômico não era garantido. No mesmo cenário, de pós Segunda Guerra Mundial, além da preocupação de estudiosos do campo econômico, a preocupação do crescimento populacional voltou a ser debatida no campo teórico demográfico.

Segundo Silva et al. (2015), a preocupação dos neomalthusianos voltava-se para o maior número de habitantes e sua implicação com a redução da renda per capita. Essa escola chegou à mesma conclusão de Malthus (1983), a de que o crescimento populacional é responsável pela miséria. Mas, diferente de Malthus (1983), que propunha soluçóes drásticas para o controle da reprodução da população, os neomalthusianos acreditam que a redução da natalidade pode ser controlada por métodos contraceptivos, e o Estado possui papel fundamental.

Os Marxistas ou Reformistas acreditavam que é a condição de pobreza que gera aumento da taxa de natalidade e elevado crescimento da população. Para melhorar o cenário são sugeridas reformas sociais e econômicas, a fim de melhorar a situaçáo dos países subdesenvolvidos, diminuir o crescimento da população e aumentar o crescimento econômico (Silva et al., 2015).

Ainda na corrente de pensamento oposta a malthusiana, encontra-se na literatura o pensamento populacionista, que trata o crescimento populacional como meio gerador de crescimento econômico. É o crescimento da população que gera dinamismo e incremento de novas tecnologias, pois os jovens são propensos a adota-las e com isso o produto da economia tende a aumentar (Stampe, 2013).

Por outro lado, nos modelos de crescimento econômico, o aumento da população foi, primeiramente, abordado por Solow (1956), com a variável força de trabalho. O modelo considera o crescimento da população como uma variável exógena. $\mathrm{Na}$ ausência de progresso tecnológico, é ela que induz o crescimento de uma economia ao estado estacionário. Portanto, a variável que aumenta o produto é a tecnologia, considerada como a força motriz do modelo. 
Nos modelos de crescimento econômico endógeno, Lucas (1988) tem o capital humano como fator impulsionador do rápido crescimento da economia. Nos modelos de crescimento lineares, Rebelo (1991) também utiliza o capital humano no progresso econômico, juntamente com o capital físico e os gastos com pesquisa. Para Romer (1990), o crescimento econômico é impulsionado pela mudança tecnológica, pelo capital humano e pela integração de mercado. $\mathrm{O}$ crescimento da população não é suficiente para o aumento do produto.

Para Bloom, Canning e Sevilla (2001), o crescimento populacional é recorrente entre os assuntos econômicos. No entanto, pouca atenção tem sido dada para a mudança na estrutura etária provocada pelo processo de transição demográfica. Para o autor, o crescimento populacional tem impacto negativo sobre o crescimento da renda per capita, porém, esse efeito pode ser contrabalanceado pelo efeito positivo do crescimento da população economicamente ativa.

As teorias econômicas e demográficas convergem em preocupação entre as interaçóes do crescimento da população e as mudanças na estrutura etária com o crescimento econômico. As investigaçóes sobre as implicaçôes da relação são tratadas por vários autores e para distintas regióes. $\mathrm{O}$ resultado comum entre eles é a relação positiva entre o número de pessoas em idade ativa e o produto per capita.

Miles (1999) mostra, através de um modelo de geraçôes sobrepostas, os impactos da transição demográfica na poupança dos indivíduos. Wong e Carvalho (2006) abordam a estrutura etária e os impactos sobre o mercado de trabalho. Lefèbvre (2006) relata os impactos da estrutura etária sobre vários indicadores macroeconômicos, como a poupança, os investimentos, o estoque de capital e o trabalho. Esses estudos levam a concluir que o processo de transição demográfica afeta o crescimento econômico por vários meios.

No âmbito internacional, Feyrer (2007) e Persson (2002) identificam a existência de relaçóes entre as variáveis demográficas e a produtividade, e indicam estudos a partir da estrutura etária. Prskawetz e Lindh (2007) e Kelley e Schimidt (2005) relacionam positivamente o crescimento econômico,o aumento da população em idade ativa e o aumento do número de jovens gera efeitos negativos sobre a renda. Para Cruz e Ahmed (2018), além da influência positiva das pessoas em idade ativa, a redução da razão de dependência dos jovens é importante para o aumento da renda per capita.

Os estudos realizados para o Brasil, como o de Porsse et al. (2012), propóem que as alteraçóes demográficas possuem implicaçóes sobre o aumento do produto no país. Stampe (2013) identifica uma relação inversa entre a razáo de dependência e o crescimento econômico, sendo que tanto a taxa de dependência de jovens quanto de idosos demonstraram influência 
negativa sobre o aumento do produto brasileiro. Entre os principais métodos utilizados encontra-se a abordagem econométrica espacial.

Estudos específicos para o Estado do Rio Grande do Sul não foram encontrados. O trabalho busca contribuir com tal lacuna na literatura. A próxima seção situa os indicadores demográficos do Rio Grande do Sul e do Brasil.

\section{A dinâmica populacional do Rio Grande do Sul/Brasil}

A América Latina possui cerca de $6 \%$ da população mundial. Segundo Brito (2007), os avanços demográficos na região latino-americana foram notórios a partir de 1950. A região latino-americana superou a áfrica no processo de transição demográfica e chegou próximo à estrutura etária da ásia. O Brasil é o país mais populoso do continente (cerca de $50 \%$ da população da América Latina) e possui a menor razão de dependência total, caracterizada por baixo número de jovens e elevado índice de idosos. Desde 1960, a menor taxa de dependência foi encontrada em 2010, a partir deste ano ela tende a aumentar, principalmente, pelo aumento de idosos na população. Em países como Peru e Uruguai o ponto de mínimo será atingido em 2030, na Bolívia e no Paraguai em 2040, e na Guiana em 2050.

A menor taxa de fecundidade da região é encontrada no Brasil em 2010, 1,80 filhos por mulher, enquanto na Bolívia o número chega a 3.2. Quanto à mortalidade infantil, a Bolívia possui 36,5 óbitos a cada mil nascidos vivos, o Chile 7.5 e o Brasil possui um indicador mediano, de 17.7 óbitos. As mesmas condiçóes são analisadas para a expectativa de vida, porém, em 2010, a menor esperança de vida da população encontrase na Guiana, seguida pela Bolívia e pelo Suriname, com 66.02, 66.40 e 70.36 anos, respectivamente.

Quanto ao crescimento da populaçáo nos estados brasileiros, nota-se que o Rio Grande do Sul possui a taxa mais baixa, em 2010 foi de $0.49 \%$. A figura 1 apresenta a taxa geométrica de crescimento anual da população residente ${ }^{1}$ dos estados brasileiros nos anos de 2000 e 2010.

A queda na taxa de crescimento da população se deve, principalmente, a queda na taxa de fecundidade. Conforme a ideia de Malthus (1983), de crescimento populacional exponencial, ao tratar do crescimento populacional dos estados brasileiros ela estaria superestimada. A taxa de crescimento da população diminui no decorrer dos anos e não assume taxa de crescimento constante, conforme o autor pressupôs. Em alguns estados, como Acre,

\footnotetext{
${ }^{1}$ A taxa geométrica de crescimento anual da população residente é obtida a partir do ano censitário imediatamente anterior. Por exemplo, o valor para o ano 2000 indica o crescimento entre 1991 e 2000 (IBGE, 2017).
} 


\section{Figura 1}

Taxa média geométrica de crescimento anual da populaçáo nos estados brasileiros em 2000 e 2010

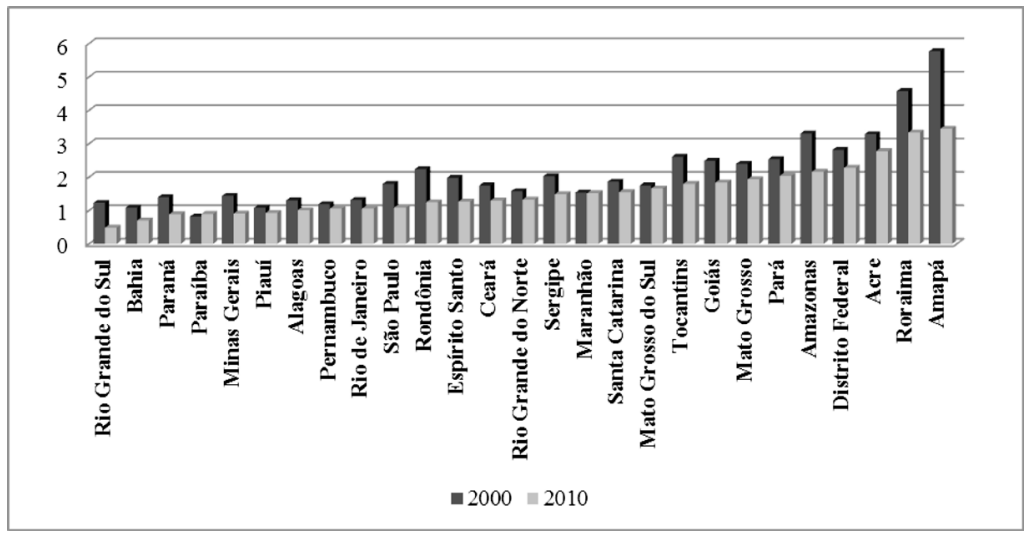

Fonte: elaborada a partir do banco de dados do Censo Demográfico do IBGE (2017).

Roraima e Amapá, o crescimento populacional ainda se encontra entre $2.50 \%$ e $3.50 \%$, maior que a taxa de crescimento mundial em 1960/70 ( $2 \%$ ao ano), quando ocorreu o seu ápice. O Rio Grande do Sul e a Bahia, de modo oposto, possuem em 2010 a taxa de crescimento projetada para a população mundial em 2040 (0.50\% ao ano) (Brito, 2007).

O Rio Grande do Sul possui no decorrer dos anos de 1991, 2000 e 2010 uma taxa de fecundidade menor que a do país, chegando a 2.38, 2.16 e 1.76, respectivamente. O estado, em 2017, possuía uma população estimada de 11,322,895 habitantes, que representa cerca de 5\% do total da população brasileira. Além disso, está entre os cinco estados mais populosos do Brasil, e a sua frente estáo Sáo Paulo, Minas Gerais, Rio de Janeiro e Bahia, com percentuais da populaçâo brasileira de $21.72 \%$, $10.17 \%, 8.05 \%$ e $7.39 \%$, respectivamente. A densidade populacional ${ }^{2}$ do Rio Grande do Sul, segundo o Censo Demográfico de 2010, é de 39 habitantes por quilômetro quadrado, assumindo a décima terceira posição no ranking dos estados brasileiros (IBGE, 2017).

O território gaúcho encontra-se dividido em 497 municípios e 35 microrregiôes. Em 2017, cerca de $85 \%$ da sua população viviam em áreas urbanas. Consequentemente, $15 \%$ estão na área rural. O estado possui características bem distintas no que tange a composição da população entre os municípios, em alguns, como Cachoeirinha, Alvorada, Canoas e Porto Alegre, toda a população é urbana, já em Chuvisca, Itati e Barão do Triunfo, mais de $90 \%$ da população é rural.

${ }^{2}$ Habitantes por quilômetro quadrado. 
Os municípios gaúchos possuem uma tendência de diminuição do número de pessoas que vivem no meio rural e aumento do percentual de pessoas em áreas urbanas. A taxa de fecundidade nos municípios do Rio Grande do Sul vem diminuindo a cada censo demográfico. No entanto, além dessa, existe outra variável relevante para a mudança no crescimento da população, a taxa de mortalidade, que apresentou uma redução entre os Censos Demográficos, de 22,71 óbitos por mil habitantes em 1991, em 2010 o número foi de 12.41 . A redução do número de óbitos gera uma população mais estável, expectativas melhores para o futuro e probabilidade de sobrevivência maior.

O Rio Grande do Sul possui um cenário demográfico distinto às demais unidades federativas do Brasil. Nesse sentido, torna-se válido o esforço em entender a sua relação com o crescimento econômico, já que a renda per capita dos municípios possui diferentes composiçóes (agrícola, industrial e serviços), bem como se difere em termos de valores absolutos entre os municípios. A próxima seção trata do método adotado na investigação.

\section{Metodologia}

Os estudos recentes trazem a análise da estrutura etária no crescimento econômico e o início da metodologia econométrica espacial. Nesta seção, a abordagem econométrica com a inclusão de defasagem espacial é caracterizada e são apresentados os modelos a serem utilizados.

\subsection{Econometria espacial}

Os modelos econométricos lineares náo levam em conta o espaço em seus parâmetros. Com isso, quando um modelo possui em seu contexto teórico e empírico a influência do espaço, o melhor método a ser utilizado é aquele em que se consegue capturar o efeito. Para isso, no modelo de regressão linear clássico (equação 1) são inseridos componentes espaciais, tais como $\boldsymbol{W} \boldsymbol{y}^{3}, \boldsymbol{W} \boldsymbol{X}^{4}, \boldsymbol{W} \boldsymbol{\xi}^{5}$ e/ou $\boldsymbol{W} \boldsymbol{\varepsilon}^{6}$.

$$
y=X \beta+\varepsilon)
$$

${ }^{3}$ Defasagem espacial da variável dependente.

${ }^{4}$ Defasagem espacial da variável independente.

${ }^{5}$ Defasagem espacial do termo de erro da regressão com alcance global. O alcance global em um modelo econométrico espacial é referenciado quando um choque em determinada variável possui impacto em seu vizinho, cuja o vizinho gera impacto em outros vizinhos e assim por diante; em que o efeito é retroalimentado chegando ao município inicial, novamente.

${ }^{6}$ Defasagem espacial do termo de erro da regressão com alcance local. $\mathrm{O}$ alcance local é modelado quando o choque de uma variável náo se propaga para todas as regióes, atingindo somente os seus vizinhos. 
Em que, $\boldsymbol{y}$ é um vetor $n$ por 1 das observaçóes da variável dependente, $\boldsymbol{X}$ é uma matriz $n$ por $k$ das variáveis explicativas exógenas, $\boldsymbol{\beta}$ é o coeficiente de regressão e $\boldsymbol{\varepsilon}$ é um vetor $n$ por 1 de termos de erro aleatórios identicamente e independentemente distribuídos (i.i.d.).

Primeiramente, o modelo é estimado via o método dos Mínimos Quadrados Ordinários (MQO), para buscar identificar qual a melhor maneira de estimar a equação 1. Alguns modelos Econométricos-Espaciais são de Defasagem Espacial: Erro Auto-Regressivo Espacial, Erro de Média Móvel Espacial, Componente de Erro Espacial, Defasagem Espacial com Erro Auto-Regressivo Espacial e Regressivo Cruzado Espacial (Almeida, 2012).

Para a identificação do modelo a ser utilizado é necessário seguir alguns procedimentos. Almeida et al. (2008) propóem a estimação do modelo via MQO e testar a hipótese de autocorrelação espacial a partir do erro ou da defasagem espacial (Multiplicador de Lagrange - $M L$ ). Se os testes não apresentarem significância, o modelo com maior ajuste é o de regressão clássico por MQO. No entanto, se os parâmetros são significativos é necessário verificar a versão robusta dos testes $(M L R)$ e analisar a significância estatística para o uso de um modelo de erro espacial ou de defasagem espacial.

A autocorrelação espacial dos resíduos da regressão é verificada por meio do teste $I$ de Moran. A estatística contempla a soma dos produtos cruzados dos resíduos para as regióes vizinhas (Almeida, 2012). A hipótese nula a ser testada é dada por:

$H_{0}$ : os resíduos da regressão são distribuídos aleatoriamente no espaço.

Para que os resíduos estejam autocorrelacionados e a técnica econométrica espacial se faça necessária, a hipótese nula deve ser rejeitada. Segundo Anselin (2005), três testes básicos devem ser utilizados para analisar a consistência dos parâmetros gerados. Sendo eles: de multicolinearidade, de normalidade dos resíduos (Jarque-Bera) e de heterocedasticidade (Breusch-Pagan, Koenker-Bassett e White). O teste de multicolinearidade não é estático, valores inferiores a 30 podem ser considerados apropriados ao modelo (Almeida, 2012). O teste Jarque-Bera para verificar a normalidade dos resíduos possui a seguinte definição:

\section{$H_{0}$ : A distribuição é normal.}

Com isto, caso a hipótese nula do teste seja rejeitada, há a evidência da distribuição dos resíduos não seguir distribuição normal. Para os testes de heterocedasticidade a hipótese a ser testada é:

$H_{0}$ : Variância constante, homocedasticidade. 
Caso a hipótese nula for rejeitada, tem-se heterocesdasticidade no modelo. Nos casos em que os resíduos não são normais e o modelo apresenta heterocedasticidade, existem alguns métodos robustos a tais problemas, como o uso de variáveis instrumentais (VI) ou por Quase Máxima Verossimilhança, em caso de um modelo com dependência espacial (SAR), e o modelo do Método dos Momentos Generalizados (MMG) quando o modelo for de defasagem do erro espacial (SEM).

A restrição de alguns parâmetros de modelagem espacial torna os modelos diferentes. Desta forma, os modelos a serem testados neste trabalho são o $\mathrm{SEM}^{7}$, o $\mathrm{SAR}^{8}$ e SARMA . A inclusão da defasagem do erro em uma forma global é testada a partir do SEM, e a defasagem do erro com aspecto local é tratada com o modelo SARMA. Isso se deve a ideia de cultura na literatura, que gera um alcance limitado entre os municípios e, também, de outras variáveis que podem gerar crescimento econômico, como as inovaçóes, as quais possuem alcance global. A defasagem da variável dependente é evidenciada tanto no modelo SAR, quanto no SARMA (Almeida, 2012).

Segundo Galor (2011) os crescimentos da renda e da população influenciam um ao outro, o que gera endogeneidade no modelo. No entanto, para Barro (1996), as variáveis geográficas (estrutura etária) são exógenas, pois depende de outras variáveis em períodos anteriores, o que leva a falta de variação no período $t$. Contudo, para prevenir problemas de endogeneidade no modelo, assim que possível, o método utilizado é o de variáveis instrumentais. Para Carvalho e Albuquerque (2010), por meio do Método do Momentos Generalizados é possível estimar o modelo com a presença de endogeneidade nas variáveis dependentes.

\subsection{Modelo espacial}

A abordagem metodológica utilizada no trabalho é baseada na especificação de Porsse et al. (2012), Stampe (2013), Prskawetz e Lindh (2007), Feyrer (2007), Weber (2010) e Cruz e Ahmed (2018). Para alcançar uma melhor compreensão da transição demográfica sobre a dinâmica econômica, Stampe (2013) atribui todos os componentes da mudança demográfica em três modelos econométricos espaciais. O primeiro, baseado na teoria de crescimento econômico de Solow (1956), leva em consideração apenas o crescimento populacional, o capital humano e a densidade de-

7 O coeficiente $\beta$ neste modelo é interpretado a partir de seu valor original, ou seja, não há um cálculo adicional de interação de $\lambda$. O efeito da mudança de $x_{k}$ sobre $y$ é diretamente captada pelo seu coeficiente econométrico, pois é resultado apenas da derivada parcial de $x_{k}$ sobre $y$.

${ }^{8}$ Após as derivaçôes cruzadas e a soma das interaçôes, Lesage e Pace (2009) relatam que os efeitos marginais totais podem decorrer da seguinte formulação: $(1-\rho)^{-1} \beta i$.

${ }^{9}$ Possui o mesmo cálculo de interação de efeitos do SAR. 
mográfica (equação 2). No segundo modelo são acrescentadas as variáveis: Razão de dependência de jovens e de idosos (equaçáo 3). O terceiro possui o mesmo conjunto de variáveis do modelo 2 , com a exclusão da RDJ e RDI, devido a multicolineariedade, e inclusão do número de pessoas em idade ativa $\left(\right.$ PIA $\left.^{10}\right)$ (equação 4).

$$
\begin{gathered}
\delta y_{i}=\beta_{0}+\rho W y_{i}+\beta_{1} \ln y_{0, i}+\beta_{2} C_{i}+\beta_{3} \ln S_{i}+\beta_{4} \ln D_{i}+W x_{0, i} \tau+\xi \\
\xi=\lambda W \xi+\varepsilon \xi \text { ou } \xi=\gamma W \varepsilon+\varepsilon
\end{gathered}
$$

$$
\begin{gathered}
\delta y_{i}=\beta_{0}+\rho W y_{i}+\beta_{1} \ln y_{0, i}+\beta_{2} C_{i}+\beta_{3} \ln S_{i}+\beta_{4} \ln D+\beta_{5} R D J_{i}+\beta_{6} R D I_{i}+W x_{0, i} \tau+\xi \\
\xi=\lambda W \xi+\varepsilon \xi \text { ou } \xi=\gamma W \varepsilon+\varepsilon
\end{gathered}
$$

$$
\begin{gathered}
\delta y_{i}=\beta_{0}+\rho W y_{i}+\beta_{1} \ln y_{0, i}+\beta_{2} C_{i}+\beta_{3} \ln S_{i}+\beta_{4} \ln D_{i}+\beta_{5} P I A+W x_{0, i} \tau+\xi \\
\xi=\lambda W \xi+\varepsilon \xi \text { ou } \xi=\gamma W \varepsilon+\varepsilon
\end{gathered}
$$

Sendo que, $\delta y_{i}$ é o logaritmo natural da taxa de crescimento da variável analisada ${ }^{11} ; \ln Y_{0, i}$ é o logaritmo da renda inicial; $C_{i}$ é o crescimento da população; $\ln S_{i}$ representa o logaritmo do nível de educação (proxy para o capital humano); $D_{i}$ é o logaritmo da densidade demográfica; $R D J_{i}$ é a razão de dependência de jovens para os $i$ municípios do RS; $R D J_{i}$ é a razão de dependência dos idosos nos municípios; PIA $A_{i}$ é $i$ a População em Idade Ativa; e $i$ representa a variável para cada município do Rio Grande do Sul. As variáveis utilizadas são melhor descritas na subseção da fonte e base de dados.

Posteriormente, conforme aborda Weber (2010) e Feyrer (2007), a análise da renda, que leva em conta apenas as razóes de dependência, é muito simples para verificar a relação entre a mudança social e o crescimento econômico. Com isso, são acrescentadas as coortes populacionais, e se especifica o modelo ${ }^{12}$ da equação 5 .

$$
\begin{gathered}
\delta y_{i}=\beta_{0}+\rho W y_{i}+\beta_{1} \ln y_{0, i}+\beta_{2} S_{i}+\beta_{3} N \eta_{i}+\beta_{4} N \theta_{i}+\beta_{5} N \mu k_{i}+\beta_{6} E_{i}+\beta_{7} D_{i}+W x_{0, i} \tau+\xi \\
\xi=\lambda W \xi+\varepsilon \xi \text { ou } \xi=\gamma W \varepsilon+\varepsilon
\end{gathered}
$$

${ }^{10}$ Pessoas que possuem entre $15-64$ anos de idade (IBGE, 2017).

${ }^{11}$ A fórmula utilizada é: $\left(\ln x_{i, t}+1-\ln x_{i, t}\right) / 19$. Como refere-se a taxa de crescimento anual, o período em estudo é de 1991 a 2010, o que compreende dezenove anos.

${ }^{12}$ Este modelo é especificado conforme Weber (2010) e Feyrer (2007). Deste modo, algumas variáveis demográficas incluídas anteriormente nas Equaçōes 2, 3 e 4, não são relevantes para esta análise, como é o caso do crescimento populacional, além do cuidado com a inclusão de variáveis que possam gerar multicolinearidade. 
Sendo que, $E_{i}$ é a expectativa de vida da populaçáa; $N \eta_{i}$ representa o número de crianças; $N \theta_{i}$ é o número de pessoas idosas; $N \mu k_{i}$ representa o número de pessoas em idade ativa de um subconjunto da populaçáo (quadro 1).

\section{Quadro 1 \\ Especificaçáo do grupo de idades para a variável $N k_{i}$}

\begin{tabular}{cc}
\hline$k_{i}$ & Grupo de idades \\
\hline 1 & $15-24$ \\
2 & $25-34$ \\
3 & $35-44$ \\
4 & $45-54$ \\
5 & $55-64$ \\
\hline
\end{tabular}

Fonte: elaborado pelos autores.

A proxy utilizada para a educação é a expectativa de anos de estudo. O modelo é calculado a partir de dados dos censos demográficos de 1991, 2000 e 2010. Segundo Stampe (2013) e Porsse et al. (2012), todas as variáveis devem representar o valor inicial de cada década no modelo de convergência de renda estudado.

\subsection{Fonte e base de dados}

Os dados utilizados para analisar o processo de transição demográfica e a sua relação com o crescimento econômico nos municípios do Rio Grande do Sul abrangem os três Censos Demográficos, de 1991, 2000 e 2010, pesquisa realizada pelo Instituto Brasileiro de Geografia e Estatística. Os dados foram extraídos do Atlas do Desenvolvimento Humano no Brasil (2017), pois a plataforma compatibiliza as informaçôes dos Censos. As variáveis utilizadas referem-se aos 496 municípios do Rio Grande do Sul, visto que o Atlas do Desenvolvimento Humano no Brasil desenvolve, sob sua metodologia de cálculo, dados para todas as variáveis utilizadas. Assim, não há problemas quanto aos municípios anteriores a 2000 não possuírem dados, já que antes desse período muitos municípios ainda não haviam sido emancipados (IBGE, 2017).

Segundo Almeida et al. (2008), as variáveis absolutas podem levar a erros na intepretação dos resultados, pois há correlação com o tamanho do município em estudo. Assim, com exceção das variáveis que já representam um percentual ou taxa, as demais encontram-se relativizada a partir do total da população no município. As variáveis utilizadas e suas especificações encontram-se no quadro 2. 


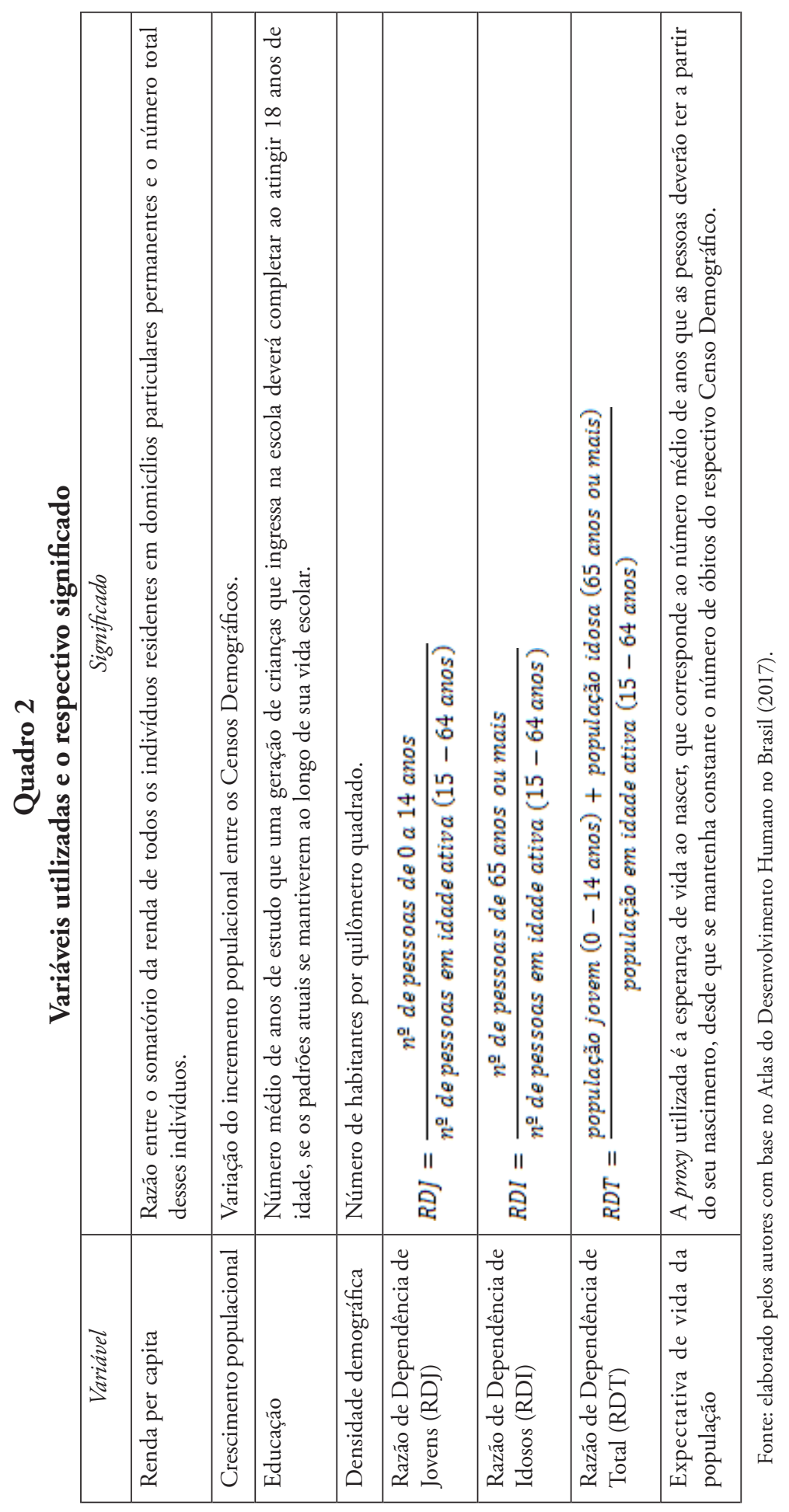


A variável renda per capita utilizada é encontrada no Atlas do Desenvolvimento Humano no Brasil ${ }^{13}$. A data de referência da deflação dos valores tem como base agosto de 2010. Com estas variáveis é possível obter resultados a fim de responder ao objetivo proposto no trabalho.

\section{Resultados e discussáo}

A importância da população para a dinâmica econômica parte de autores clássicos na literatura, como Malthus (1983), Marshall (1996) e Kuznets (1986). Dentro dos modelos de crescimento econômico, a proposta de Solow (1956) trata do crescimento absoluto da população. Como base para outras abordagens, passa-se a acrescentar mais variáveis no modelo. As equaçóes 2 e 3 partem deste enfoque ${ }^{14}$, e seu resultado para os municípios do Rio Grande do Sul pode ser visualizado na tabela 1.

Para Almeida (2012), um valor condicional acima de 110 representa um problema, assim, o valor apresentado no modelo é adequado. Como os resíduos da regressão não são normais e ocorre o problema de heterocedasticidade, optou-se por estimar o modelo SEM e o SARMA, ambos significativos nas duas especificações, pelo método dos momentos generalizados de Kelejian e Prucha (1999) com erro heterocedástico. A adoção do método não necessita de normalidade dos resíduos e é possível acomodar a ausência de homocedasticidade por meio de grupos.

Como forma de garantir a especificação adequada, devido à imbricação entre heterocedasticidade e autocorrelação espacial, foram testados outros modelos, em que o coeficiente espacial do erro continuou significativo, mesmo quando a defasagem da variável dependente não apresentou significância. Não há diferenças consideráveis entre os coeficientes dos dois modelos (MQO e SEM/SARMA), todos os modelos e métodos testados convergem para o mesmo resultado.

A convergência da renda per capita entre o ano de 1991 e 2010 é significativa, com efeitos espaciais ou sem eles, o que muda entre os dois é o grau de convergência. Na equação 2, com a introdução dos efeitos espaciais, o processo de convergência é mais rápido. Na equação 3 existe o efeito direto e indireto da variável dependente do vizinho na renda de

${ }^{13} \mathrm{O}$ cálculo da variável parte de métodos como: exclusão, hot desk probabilístico, hot desk determinístico, imputação múltipla, discriminante e vizinhos mais próximos. A utilização destes métodos decorre do cuidado com as variáveis faltantes no cálculo da renda per capita, o que pode levar a viés da análise.

${ }^{14}$ A estimação da equação 4 gerou o problema de multicolineariedade, como a estrutura etária encontra-se na equação 5 , optou-se por não a analisar. 


\section{Tabela 1}

Análise de convergência das equaçóes 2 e 3 condicionadas à variáveis demográficas, 1991/2010

\begin{tabular}{|c|c|c|}
\hline & Equação (2) & Equação (3) \\
\hline Coeficientes & $1991 / 2010$ & $1991 / 2000$ \\
\hline Constante & $\begin{array}{r}3,83204 \\
(0,00000)\end{array}$ & $\begin{array}{r}4,81844 \\
(0,00000)\end{array}$ \\
\hline \multirow[t]{2}{*}{ Renda Inicial } & $-0,52071$ & $-0,620685$ \\
\hline & $(0,00000)$ & $(0,00000)$ \\
\hline \multirow[t]{2}{*}{ Crescimento da População } & $-0,127217$ & $-0,0946349$ \\
\hline & $(0,00331)$ & $(0,02690)$ \\
\hline \multirow[t]{2}{*}{ Educação } & 0,00432773 & 0,045197 \\
\hline & $(0,94074)$ & $(0,42226)$ \\
\hline \multirow[t]{2}{*}{ Densidade Demográfica } & 0,0000324 & 0,000007 \\
\hline & $(0,39483)$ & $(0,05924)$ \\
\hline \multirow[t]{2}{*}{ RDJ } & & $-1,06869$ \\
\hline & & $(0,00000)$ \\
\hline \multirow[t]{2}{*}{ RDI } & & $-0,46778$ \\
\hline & & $(0,61413)$ \\
\hline AIC & $-144,643$ & $-184,247$ \\
\hline SC & $-123,61$ & $-154,801$ \\
\hline \multirow[t]{2}{*}{$I$ de Moran } & 13,6042 & 13,4265 \\
\hline & $(0,00000)$ & $(0,00000)$ \\
\hline \multirow[t]{2}{*}{ ML - Erro } & 176,0160 & 169,0566 \\
\hline & $(0,00000)$ & $(0,00000)$ \\
\hline \multirow[t]{2}{*}{ ML - Defasagem } & 78,2600 & 107,0979 \\
\hline & $(0,00000)$ & $(0,00000)$ \\
\hline \multirow[t]{2}{*}{ MLR - Erro } & 99,9826 & 63,2707 \\
\hline & $(0,00000)$ & $(0,00000)$ \\
\hline \multirow[t]{2}{*}{ MLR - Defasagem } & 2,2266 & 1,3120 \\
\hline & $(0,13565)$ & $(0,25203)$ \\
\hline \multirow[t]{2}{*}{ SARMA } & 178,2426 & 170,3686 \\
\hline & $(0.00000)$ & $(0,00000)$ \\
\hline Teste Breusch- & 12,5161 & 16,4050 \\
\hline Pagan & $(0,01390)$ & $(0,01174)$ \\
\hline \multirow[t]{2}{*}{ Teste Jarque-Bera } & 24,7457 & 25,6137 \\
\hline & $(0,00000)$ & $(0,00000)$ \\
\hline Condition Number & 43,418278 & 73,4721222 \\
\hline
\end{tabular}

Fonte: elaborada pelos autores com base nos dados do Censo Demográfico de 1991, 2000 e 2010. 
cada município, o que contribui para diminuir ${ }^{15}$ o processo de convergência $(-0,53$ com efeitos de $W y)$ (tabela 2$)$.

Com a adequação do modelo e do método utilizado, é possível inferir que variáveis como a densidade demográfica e a razão de dependência dos jovens são altamente significativas nas duas especificaçôes propostas. $\mathrm{O}$ aumento da densidade demográfica e a redução da razão de dependência de jovens contribuem para o aumento da renda per capita. O crescimento econômico e da população possuem uma relação inversa ${ }^{16}$ na equação 2 , o mesmo sinal esperado no modelo de crescimento de Solow (1956). Porém, com a inclusão de variáveis que compóem a estrutura etária da população (RDT e RDI), o crescimento absoluto da mesma não é significativo.

Os estudos empíricos para analisar o papel da estrutura etária sobre o aumento do produto tiveram seu início, principalmente, na década de 1990. Barro (1996) em seu estudo envolvendo o crescimento econômico de cem países, foi um dos pioneiros a introduzir um conjunto de variáveis demográficas. Neste foi evidenciada a relação inversa entre o produto per capita e as taxas de fertilidade, de crescimento da população e de mortalidade, ou a relaçáo positiva entre a renda e a densidade populacional. Os estudos de Barro (1996 e 2007) contribuem para a interpretaçáo dos resultados nos municípios do Rio Grande do Sul, uma vez que um aumento da densidade demográfica também gera um aumento do PIB per capita, o que pode ocorrer devido ao aumento de pessoas em idade ativa ou a concentração industrial (áreas dinâmicas que induzem a imigração).

Com a inclusão da estrutura etária (equação 3), a razão de dependência de jovens é negativa e significativa, ou seja, quando o número de jovens é elevado frente à população em idade ativa, o produto per capita tende a ser menor. A PIA possui mais dependentes para sustentar, o que, consequentemente, diminui a renda per capita. Esse resultado está em acordo com a literatura, pois ao analisar o crescimento econômico e a estrutura etária na União Europeia, Prskawetz e Lindh (2007) concluem que independentemente do método utilizado, a variável razão de dependência de jovens é robusta nos modelos e possui impacto significativo e negativo.

Neste sentido, Stampe (2013) utilizou um modelo de dados em painel para a análise das regiôes estatisticamente comparáveis do Brasil. O estudo evidenciou a relação inversa entre a razão de dependência e o crescimento econômico, sendo que tanto a taxa de dependência de jovens quanto de idosos demonstraram influência negativa sobre o aumento do produto brasileiro. Resultado semelhante foi encontrado por Cruz e Ahmed (2018),

\footnotetext{
${ }^{15}$ Parâmetro negativo indica dissimilaridade.

${ }^{16}$ Com significância estatística a $10 \%$.
} 


\section{Tabela 2}

Análise de convergência das equaçóes 2 (SEM) e 3 (SARMA*) condicionadas à variáveis demográficas, 1991/2010

\begin{tabular}{|c|c|c|}
\hline & Equaçâa (2) & Equação (3) \\
\hline Coeficiente & $1991 / 2010$ & $1991 / 2000$ \\
\hline Constante & $\begin{array}{r}4,2617785 \\
(0,00000)\end{array}$ & $\begin{array}{r}4,9453277 \\
(0,00000)\end{array}$ \\
\hline \multirow[t]{2}{*}{$\lambda$} & 0,6006850 & \\
\hline & $(0,00000)$ & \\
\hline \multirow[t]{2}{*}{$\gamma$} & & 0,5226605 \\
\hline & & $(0,00000)$ \\
\hline \multirow[t]{2}{*}{$\rho$} & & $-0,1340171$ \\
\hline & & $(0,09673)$ \\
\hline \multirow[t]{2}{*}{ Renda Inicial } & $-0,6072403$ & $-0,6748744$ \\
\hline & $(0,00000)$ & $(0,00000)$ \\
\hline \multirow[t]{2}{*}{ Crescimento da População } & $-0,0729861$ & $-0,0330589$ \\
\hline & $(0,09262)$ & $(0,44163)$ \\
\hline \multirow[t]{2}{*}{ Educação } & $-0,0414382$ & $-0,0274054$ \\
\hline & $(0,5118925)$ & $(0,65867)$ \\
\hline \multirow[t]{2}{*}{ Densidade Demográfica } & 0,0506487 & 0,0667741 \\
\hline & $(0,00003)$ & $(0,00000)$ \\
\hline \multirow[t]{2}{*}{ RDJ } & & $-1,1701196$ \\
\hline & & $(0,00000)$ \\
\hline \multirow[t]{2}{*}{ RDI } & & 0,6884503 \\
\hline & & $(0,45454)$ \\
\hline
\end{tabular}

* A variável taxa de crescimento da renda per capita foi instrumentada pela defasagem especial das variáveis: crescimento da população, densidade demográfica, educação, renda inicial, RDI e RDJ.

Fonte: elaborada pelos autores com base nos dados do Censo Demográfico de 1991, 2000 e 2010.

a redução da dependência de jovens contribui para o aumento do Produto Interno Bruto dos países.

Porsse et al. (2012) analisaram a convergência do crescimento econômico em uma perspectiva regional a partir de variáveis demográficas. $\mathrm{O}$ estudo buscou identificar, através de um modelo de dados em painel espacial, a influência da mudança na estrutura etária para a convergência de renda dos estados brasileiros, nos anos de 1970, 1980, 1991 e 2000. Os autores verificaram que as alteraçóes demográficas possuem implicação sobre o aumento do produto e a convergência do mesmo, ao passo que a 
razão de dependência de jovens possui relação negativa e a de idosos positiva.

O modelo baseado em Solow (1956), sem a inclusão da estrutura etária, apresenta dependência espacial no erro, ou seja, é um choque de variáveis não modeladas que exibem padrão espacial. Na inclusão da RDJ e RDI, além do erro espacial, a defasagem espacial da renda inicial foi significativa a $10 \%$ e negativa, indicando que existe transbordamento espacial, porém há dissimilaridade da renda nos municípios. A inclusão de um vetor de variáveis que representa as coortes populacionais pode contribuir para a verificação da origem da dependência espacial, bem como com a investigação da relação das variáveis demográficas e o crescimento da renda per capita.

Feyrer (2007) realizou um estudo da relação demográfica e a produtividade agregada, através de um modelo para alguns países, em que verificou a existência de correlaçóes ${ }^{17}$ entre as variáveis. A mesma conclusão foi evidenciada por Persson (2002) em um trabalho realizado para os Estados Unidos entre 1930 e 2000. Feyrer (2007) relata a importância das pesquisas realizadas por Barro (1996 e 2007) e Lee (2006), que tratam da relevância da estrutura social e não apenas a taxa de crescimento da população ou o tamanho da força de trabalho, como nos modelos neoclássicos.

Para Weber (2010), o conjunto de variáveis desagregadas por níveis de idade é importante para verificar todasas características da população sobre o crescimento econômico. A tabela 3 traz a análise da desagregação da PIA em cinco grupos de idade, de 15 a 24 anos, 25 a 34 anos, 35 a 44 anos, 45 a 54 anos e 55 a 64 anos.

Com a inclusão do vetor de coortes populacionais o valor do parâmetro de convergência espacial aumentou, tanto sem a inclusão da interação do espaço quanto com ela, $-0.78 \mathrm{e}-0.94$, respectivamente. Isso indica que o crescimento econômico é influenciado pela estrutura etária da população ${ }^{18}$. Neste modelo, a inclusão da variável dependente defasada é positiva e significativa, o que indica transbordamento espacial, e a variação da renda per capita dos municípios vizinhos é um determinante importante para a variação da renda no estado.

O nível de educação, o percentual de idosos e de jovens nos municípios, não possuem significância estatística. Com isso, a análise da sua relação

\footnotetext{
17 "While this paper shows that there is a relationship between productivity and demographics, more research is needed to understand the mechanisms behind this relationship." (Feyrer, 2007).

${ }^{18}$ A equaçáo 5 foi testada sem os vetores de jovens e idosos, para evitar problemas de multicolineariedade. Com isso, o vetor de coortes populacionais, a renda inicial, a densidade demográfica e a esperança de vida da populaçáo foram analisadas. Os parâmetros náo tiveram alteraçóes significativas, e optou-se por manter o modelo original, proposto por Feyrer (2007).
} 


\section{Tabela 3}

Análise de convergência da equaçáo 5 condicionada à estrutura etária, 1991/2010

\begin{tabular}{|c|c|c|}
\hline & $M Q O$ & $S A R M A^{1}$ \\
\hline Coeficientes & $1991 / 2010$ & $1991 / 2010$ \\
\hline Constante & $\begin{array}{r}1,2542 \\
(0,00090)\end{array}$ & $\begin{array}{r}1,3499422 \\
(0,00050)\end{array}$ \\
\hline$\gamma$ & & $\begin{array}{l}0,2244452 \\
(0,00296)\end{array}$ \\
\hline$\rho$ & & $\begin{array}{r}0,2248647 \\
(0,00003)\end{array}$ \\
\hline Renda Inicial & $\begin{array}{r}-0,786711 \\
(0,00000)\end{array}$ & $\begin{array}{r}-0,7317191 \\
(0,00000)\end{array}$ \\
\hline Educação & $\begin{array}{r}-0,0539019 \\
(0,25921)\end{array}$ & $\begin{array}{r}-0,0919680 \\
(0,12947)\end{array}$ \\
\hline Jovens & $\begin{array}{l}-0,51074 \\
(0,14391)\end{array}$ & $\begin{array}{c}-0,3752336 \\
(0,22736)\end{array}$ \\
\hline Idosos & $\begin{array}{l}-1,22441 \\
(0,30803)\end{array}$ & $\begin{array}{c}-0,3115972 \\
(0,78066)\end{array}$ \\
\hline PIA $15-24$ & $\begin{array}{l}0,710355 \\
(0,33735)\end{array}$ & $\begin{array}{r}0,4967974 \\
(0,54203)\end{array}$ \\
\hline PIA $25-34$ & $\begin{array}{r}1,45266 \\
(0,02977)\end{array}$ & $\begin{array}{r}2,0404505 \\
(0,00402)\end{array}$ \\
\hline PIA $35-44$ & $\begin{array}{r}7,58218 \\
(0,00000)\end{array}$ & $\begin{array}{r}6,7554513 \\
(0,00000)\end{array}$ \\
\hline PIA $45-54$ & $\begin{array}{r}-0,144602 \\
(0,89164)\end{array}$ & $\begin{array}{r}0,2176232 \\
(0,84571)\end{array}$ \\
\hline PIA $55-64$ & $\begin{array}{r}2,17738 \\
(0,01401)\end{array}$ & $\begin{array}{r}2,8115173 \\
(0,00280)\end{array}$ \\
\hline ESP. VIDA & $\begin{array}{r}0,0406082 \\
(0,00000)\end{array}$ & $\begin{array}{r}0,0316699 \\
(0,00000)\end{array}$ \\
\hline Densidade Demográfica & $\begin{array}{r}0,0431851 \\
(0,00000)\end{array}$ & $\begin{array}{r}0,0417352 \\
(0,00007)\end{array}$ \\
\hline AIC & $-357,049$ & \\
\hline SC & $-306,57$ & \\
\hline$I$ de Moran & $\begin{array}{r}8,2319 \\
(0,00000)\end{array}$ & \\
\hline ML - Erro & $\begin{array}{r}61,3449 \\
(0,00000)\end{array}$ & \\
\hline
\end{tabular}


Tabela 3 (continua)

\begin{tabular}{lrr}
\hline \multicolumn{1}{c}{ Coeficientes } & \multicolumn{1}{c}{ MQO } & \multicolumn{1}{c}{ SARMA ${ }^{1}$} \\
\hline ML - Defasagem & $1991 / 2010$ & \\
MLR - Erro & 56,2162 & \\
& $(0,00000)$ & \\
MLR - Defasagem & 17,0006 & \\
& $(0,00004)$ & \\
SARMA & 11,8719 & \\
\hline & $(0,00057)$ & \\
\hline Teste Breusch- & 73,2168 & \\
Pagan & $(0.00000)$ & \\
Teste Jarque-Bera & 49,0411 & \\
& $(0,00000)$ & \\
\hline
\end{tabular}

Fonte: elaborada pelos autores com base nos dados do Censo Demográfico de 1991, 2000 e 2010.

com o crescimento náo é realizada. $\mathrm{O}$ percentual de pessoas entre as faixas etárias de 15 a 24 anos e de 45 a 54 anos, também não apresentaram significância. Para Paschoal (2008), até os 23 anos, muitos jovens ainda estão nas universidades, o que pode contribuir para o resultado obtido. No estudo realizado por Feyrer (2007), algumas coortes também não apresentaram significância, enquanto as pessoas entre 35 a 45 anos são as que mais contribuem para o crescimento econômico.

As pessoas entre 35 e 45 anos foram as que mais contribuíram para o aumento da produção per capita do Rio Grande do Sul. Seguida da população com 55 a 64 anos e, por último, dos indivíduos entre 25 e 34 anos. Prskawetz e Lindh (2007), Kelley e Schimidt (2005), os quais estudaram a Europa, encontraram uma relação do aumento do produto com a estrutura etária da população, a qual assume uma curva em formato de "U" invertido, ou seja, é na idade de 30 a 49 anos que a população possui maior influência sobre o crescimento econômico.

A inclusão da variável que representa a expectativa de vida da população (esperança de vida ao nascer) traz contribuiçóes importantes para a análise, visto que, a melhoria na saúde e na qualidade de vida da população são os principais meios de aumento do ciclo de vida. A ideia proposta pelo FMI (2016) e pela UNFPA (2016) ganha evidências empíricas: o estado de saúde da população é um importante meio de aumento da produtividade e, com isso, do aproveitamento do bônus demográfico.

Ao estudar a transição demográfica e os desafios para o crescimento econômico brasileiro, Pereima e Porsse (2013) preocupam-se com a ace- 
leração da mudança na estrutura etária e as implicaçôes do fim do bônus demográfico, o que gera mudança na demanda efetiva, no consumo, na poupança e na capacidade de crescimento da economia. No momento em que a razáo de dependência de idosos chega a $8 \%$ da populaçáo total, o crescimento econômico não pode ser sustentado apenas pela acumulação de capital. Nesta fase, o crescimento econômico passa por algumas restriçóes e depende do aumento da produtividade e da invenção de novos produtos.

Bloom et al. (2009) e o FMI (2016) relatam a importância da mulher no mercado de trabalho. Em meio a transição para o envelhecimento da população, com níveis educacionais relevantes, elas podem ser as futuras promotoras do crescimento econômico. Feyrer (2007) introduziu na literatura um modelo demográfico de crescimento endógeno, diferenciando a mão de obra qualificada da que possui pouca qualificação, o que resultou em importantes consequências para o crescimento. Com a diminuição de mão de obra no mercado de trabalho, algumas fontes alternativas devem ser propostas, e uma das alternativas é a mão de obra da população feminina.

\section{Conclusão}

A dinâmica populacional é um processo amplo, com reflexos em variáveis econômicas e sociais. O objetivo deste estudo foi analisar as mudanças na estrutura etária e a sua relaçáo com o crescimento econômico nos municípios do Rio Grande do Sul, a partir dos Censos Demográficos de 1991, 2000 e 2010.

Com base no modelo de crescimento de Solow (1956), identificou-se que o crescimento da populaçáo e o crescimento da renda possuem relação inversa. No estudo, verificou-se que o aumento da densidade demográfica gera aumento do PIB per capita. Avançando na estrutura dos modelos de crescimento, com inclusão de variáveis demográficas com maior amplitude, como as razóes de dependência, pode-se verificar que nos municípios gaúchos quando o número de jovens é elevado, ao comparar com a população em idade ativa, o produto per capita tende a ser menor.

Com a inclusão de coortes populacionais na análise, a convergência da renda aumentou, sendo que as pessoas com idade entre 35 a 45 anos são as que mais contribuem para o crescimento econômico, seguidas da população entre 55 e 64 anos e de 25 a 34 anos de idade. A variável esperança de vida ao nascer, que representa a expectativa de vida, foi positiva e significativa. $\mathrm{O}$ 
resultado é importante, pois a variável pode representar o estado de saúde e a qualidade de vida do indivíduo, que quanto melhor, maior é a produtividade.

Essa última evidência abre questóes para outros estudos a serem realizados, que devem ir além da ótica econômica do crescimento do produto por meio de variáveis clássicas. Cabe ressaltar, que o crescimento econômico vai além da dinâmica demográfica, as variáveis aqui apresentadas se referem, especificamente, a atender o objetivo proposto. Outra forma de aumento da renda é aproveitar a mão de obra das mulheres, que está entre as mais qualificadas do mercado de trabalho. Também, podem ser incorporadas variáveis com relação a taxa de desemprego por idade ou a formalizaçáo do emprego. Contudo, os resultados deste trabalho podem contribuir para políticas públicas e sociais, em que o crescimento da população e o envelhecimento dela não sejam considerados um obstáculo para o crescimento da economia.

\section{Referências}

Almeida, Eduardo Simóes, Fernando Perobelli Salgueiro e Pedro Guilherme Ferreira Costa (2008), "Existe convergência espacial da produtividade agrícola no Brasil?", Revista de Economia e Sociologia Rural, 46 (1), Libri Laboris, Rio de Janeiro Brasil, p.22.

Almeida, Eduardo (2012), Econometria Espacial Aplicada, 1. Ed., Editora Alínea, Campinas, Brasil.

Anselin, Luc (2005), Exploring Spatial Data with GeoDa: a Workbook, University of Illinois, Urbana-Champaign-Ed. Copyright, Chicago, Estados Unidos da América.

Atlas do Desenvolvimento Humano no Brasil (2017), "O Atlas", Atlas do Desenvolvimento Humano no Brasil, Rio de Janeiro, Brasil, <http://www.atlasbrasil.org.br/2013/pt/o_atlas/idhm/>, 16 do abril do 2017.

Barro, Robert (2007), "Economic Growth in a Cross Section of Countries”, Quarterly Journal of Economics, 106 (2), The MIT Press, Cambridge, Inglaterra, pp. 407-444.

Barro, Robert (1996), Determinants of Economic Growth: A Cross-Country Empirical Study, MIT: Press, Cambridge, Inglaterra. 
Bloom, David, David Canning, Gunther Fink e Jocelyn Finlay (2009), "Fertility, female labor force participation, and the demographic dividend. Journal of Economic Growth", Springer Science Business Media, 14 (2), Harvard University, Boston, Estados Unidos da América, pp. 79-101.

Bloom, David, David Canning e Jaypee Sevilla (2001), Economic Growth and the demographic transition, National Bureau of Economic Research, Cambridge, Inglaterra.

Brito, Fausto (2008), "Transição demográfica e desigualdades sociais no Brasil”, Revista Brasileira de Estudos da População, 25 (1), Associação Brasileira de Estudos Populacionais, São Paulo, Brasil, pp. 5-26.

Brito, Fausto (2007), "A transição demográfica no contexto internacional”, texto para discussáo núm. 317, Centro de Desenvolvimento e Planejamento Regional da Universidade Federal de Minas Gerais, Minas Gerais, Brasil.

Carvalho, Alexandre Xavier Ywata e Pedro Henrique Albuquerque Melo (2010), "Tópicos em econometria espacial para dados cross-section", num. 1508, Instituto de Pesquisa Econômica Aplicada, Brasília, Brasil.

Cruz, Marcio e Amer Ahmed (2018), "On the impact of demographic change on economic growth and poverty", World Development, 105, Elsevier, Amsterdam, Holanda, pp. 95-109.

Feyrer, James (2007), “Demographics and Productivity. Dartmouth College Working Paper", Review of Economics and Statistics, 89 (1), Dartmouth College, Hanover, Estados Unidos da América, pp. 100-109.

FMI (Fundo Monetário Internacional) (2016), "Data”, FMI, Santa Maria, Brasil, <http://www.imf.org/external/index.htm>, 12 do novembro do 2016.

Galor, Oded (2011), Teoria do crescimento unificado, Princeton University Press, Princeton, Estados Unidos da América.

IBGE (Instituto Brasileiro de Geografia e Estatística) (2017), "PIMPFProdução física industrial por seçôes e atividades industriais", 
IBGE, Rio de Janeiro, Brasil, <http://www.sidra.ibge.gov.br>, 18 do fevereiro do 2017.

Kelejian, Harry e Ingmar Prucha (1999), "A Generalized Moments Estimator for the Autoregressive Parameter in a Spatial Model", Revisão econômica internacional, 40 (2), Wiley Online Library, Pensilvânia, Estados Unidos da América, pp. 509-533.

Kelley, Allen e Robert Schmidt (2005), "Evolution of recent economicdemographic modeling: A synthesis", Journal of Population Economics, 18 (2), Springer-Verlag, Los Angeles California, Estados Unidos da América, pp. 275-300.

Kuznets, Simon (1986), Crescimento econômico moderno: ritmo, estrutura e difusão, Abril Cultural, São Paulo, Brasil.

Lee, Ronald (2006), "The demographic transition: Three centuries of fundamental change", Journal of Economic Perspectives, 17 (4), American Economic Association, New Haven, Estados Unidos da América, pp. 167-190.

Lefèbvre, Mathieu (2006), Population ageing and consumption demand in Belgium, Crepp, Liège, Bélgica.

Lesage, James e Robert Kelley Pace (2009), Modelos econométricos espaciais. Manual de análise espacial aplicada, Springer, Berlin, Alemania.

Lewis, Arthur (1969), "O desenvolvimento econômico com oferta ilimitada de mão-de-obra”, A Economia do Subdesenvolvimento, 1 (1), Ed. Forense, Rio de Janeiro, Brasil, pp. 406-456.

Lucas, Robert Júnior (1988), "On the mechanics of economic development", Journal of Monetary Economics, 22 (1), Elsevier, Amsterdam, Holanda, pp. 3-42.

Malthus, Thomas Robert (1983), Ensaio sobre a população vol. 328, Abril Cultural, São Paulo, Brasil.

Marshall, Alfred (1996), Princípios de Economia, vol. 1, Editora Nova Cultural, São Paulo, Brasil. 
Miles, David (1999), "Modelling the Impact of Demographic Change upon the Economy", The Economic Journal, 109 (452), Blackwell Publishers, Malden, Estados Unidos da América, pp. 1 - 36.

ONU (Organização das Nações Unidas) (2017), "Base de dados”, ONU, Santa Maria, Brasil, < https://nacoesunidas.org/>, 14 do novembro do 2017.

Paschoal, Isabela Palma (2008), "Mobilidade intergeracional de educação no Brasil", dissertação mestrado, Faculdade de Economia, Administraçáo e Contabilidade de Ribeirão Preto, Universidade de São Paulo, Ribeirão Preto, Brasil.

Pereima, João Basilio e Alexandre Porsse (2013), “Transição demográfica, acumulação de capital e progresso tecnológico: desafios para o crescimento brasileiro", Revista Economia and Tecnologia, 9 (1), Universidade Federal do Paraná, Curitiba, Brasil, pp. 49-60.

Persson, Joakin (2002), Demographics, human capital, and economic growth: a study of US States 1930-2000, FIEF, Estocolmo, Suécia.

Prskawetz, Alexia e Thomas Lindh (2007), “The relationship between demographic change and economic growth in the EU", report núm. 32, Vienna Institute of Demography, Research, Viena, Austria.

Porsse, Alexandre, Marianne Stampe, Marcelo Portugal e Eduardo Almeida (2012), Demographic Change and Regional Economic Growth in Brazil, Regional and Urban Economics Lab, University of São Paulo, São Paulo, Brasil.

Rebelo, Sergio (1991), "Long run policy analysis and long run growth", Journal of Political Economy, 99 (3), The University of Chicago Press, Chicago, Estados Unidos da América, pp. 500-521.

Romer, Paul (1990), "Endogenous Technological Change”, Journal of Political Economy, 98 (5), The University of Chicago Press Journals, Chicago, Estados Unidos da América, pp. 71-102.

Silva, José Adailton Barroso, Raphael Luiz Fontana Macêdo, Silvania Costa Santana e Auro Jesus Rodrigues (2015), "Teorias demográficas e o crescimento populacional no mundo", Caderno de 
Graduação-Ciências Humanas e Sociais-UNIT, 2 (3), Grupo Tiradentes, Aracaju, Brasil, pp. 113-124.

Solow, Robert (1956), "Contribution to the theory of economic growth", Quarterly Journal of Economics, vol. 70, Oxford Academic, Oxfordshire, Inglaterra, pp. 65-94.

Stampe, Marianne (2013), “Três ensaios sobre mudança demográfica e seus impactos nas economias brasileira e gaúcha”, tese de doutorado, Universidade Federal do Rio Grande do Sul, Rio Grande do Sul, Brasil.

UNFPA (United Nations Population Fund) (2016), "How our future depends on girls at this decisive age", UNFPA, Nova York, Estados Unidos da América, <https://www.unfpa.org/sites/default/ files/pubpdf/The_State_of_World_Population_2016_-_English. pdf>, 23 do agosto do 2017.

Vasconcelos, Daniel Santana, José Eustáquio Alves Diniz e Getúlio Silveira filho Borges (2008), "Crescimento Econômico, estrutura etária e dividendo demográfico: avaliando a interaçáo com dados em painel", XVI Encontro Nacional de Estudos Populacionais da ABEP, 29 de setembro a 3 de outubro, Minas Gerais, Brasil.

Weber, Lars (2010), Demograpfhic change and economic growth: simulations and growth models, London, Nova York: Springer Heidelberg Dordrecht, Inglaterra.

Wong, Laura Rodrigues e José Alberto Carvalho (2006), “O rápido processo de envelhecimento populacional do Brasil: sérios desafios para as políticas públicas", Revista Brasileira de Estudos Populacionais, 23 (1), Associação Brasileira de Estudos Populacionais, Minas Gerais, Brasil, pp. 5-26.

Recibido:13 de marzo de 2018. Revisado: 15 de junio de 2018. Aceptado: 13 de julio de 2018.

Lauana Rossetto Lazaretti. Doutoranda em Economia pela Pontifícia Universidade Católica do Rio Grande do Sul. Atualmente é bolsista-pesquisadora da Coordenação de Aperfeiçoamento de Pessoal de Nível Su- 
perior (CAPES). Sua linha de pesquisa atual é a transição demográfica no Brasil. Entre as suas últimas publicaçôes, destacam-se: "Os estados brasileiros na transição demográfica: similaridades e características discriminantes", PRACS: Revista Eletrônica de Humanidades do Curso de Ciências Sociais da UNIFAP, 2 (10), Universidade Federal do Amapá, Macapá, Brasil, pp. 9-24 (2017), "Efeitos socioeconômicos da transição demográfica nos estados brasileiros: uma análise de clusters", Observatorio de la Economia Latinoamericana, vol. 233, Servicios Académicos Intercontinenetales S.L.-GrupoEUMED.NET, Malaga, España, pp. 1-15 (2017) e "Crescimento Econômico restrito pelo balanço de pagamentos e a evolução das exportações brasileiras: uma análise através da Lei de Thirlmall”, Estudos do CEPE, num. 46, Universidade Santa Cruz do Sul, Santa Cruz do Sul, Brazil, pp. 39-57 (2017).

Pascoal José Marion Filho. Doutor em Economia Aplicada pela Escola Superior de Agricultura Luiz de Queiroz da Universidade de São Paulo - (Esalq/USP). Atualmente é professor titular do Programa de Pós-Graduação em Economia e Desenvolvimento e do Programa de Pós-Graduação em Administração da Universidade Federal de Santa Maria, Rio Grande do Sul, Brasil. Sua linha de pesquisa atual é a transição demográfica no Brasil e a cadeia global de valor. Entre suas últimas publicaçóes destacam-se: "Transição demográfica no Rio Grande do Sul: um processo desafiador", Revista de Desenvolvimento Regional em debate, 1(7), Universidade do Contestado, Mafra, Brasil, pp. 196-213, (2017), "Condicionantes econômicos e sociais da fecundidade no Brasil", Revista de Ciências Sociais em Perspectiva, 30 (16), Universidade Estadual do Oeste do Paraná, Paraná, Brasil, pp. 39-57 (2017) e "Os estados brasileiros na transição demográfica: similaridades e características discriminantes”, PRACS: Revista Eletrônica de Humanidades do Curso de Ciências Sociais da UNIFAP, 2) (10), Universidade Federal do Amapá, Macapá, Brasil, pp. 9-24 (2017). 